\title{
Measurements of electron cloud density in the CERN Super Proton Synchrotron with the microwave transmission method
}

\author{
S. Federmann, F. Caspers, and E. Mahner \\ CERN, 1211 Geneva 23, Switzerland \\ (Received 19 May 2010; published 24 January 2011)
}

\begin{abstract}
The electron cloud effect can pose severe performance limitations in high-energy particle accelerators as the CERN Super Proton Synchrotron (SPS). Mitigation techniques such as vacuum chamber thin film coatings with low secondary electron yields (SEY $<1.3$ ) aim to reduce or even suppress this effect. The microwave transmission method, developed and first applied in 2003 at the SPS, measures the integrated electron cloud density over a long section of an accelerator. This paper summarizes the theory and measurement principle and describes the new SPS microwave transmission setup used to study the electron cloud mitigation of amorphous carbon coated SPS dipole vacuum chambers. Comparative results of carbon coated and bare stainless steel dipole vacuum chambers are given for the beam with nominal LHC 25 ns bunch-to-bunch spacing in the SPS and the electron cloud density is derived.
\end{abstract}

DOI: 10.1103/PhysRevSTAB.14.012802

PACS numbers: 29.27.Bd, 29.20.dk

\section{INTRODUCTION}

Inside the vacuum chamber of high-energy particle accelerators a small number of electrons are always present and unavoidable. The presence of electrons can be caused, for example, by ionization of residual gas molecules, by desorption of lost beam particles, and by photoemission due to synchrotron radiation. These electrons, once accelerated by the beam potential, impact on the vacuum chamber wall and create secondary electrons, which can lead to an avalanche creating a so-called electron cloud (EC).

This EC effect can be detrimental for the performance of a particle accelerator, leading to significantly fast and high pressure rises, beam instabilities such as transverse blowup and particle losses resulting in a limitation of the beam intensity, and a reduction of beam quality. At CERN, efforts are presently focused on the production, characterization, and implementation of thin films with low secondary electron yields $(\mathrm{SEY}<1.3)$ due to its potential application in future accelerator upgrade programs, as the CERN Super Proton Synchrotron (SPS) upgrade for LHC, but also for the design of new machine vacuum systems, for example the PS2 and the CLIC damping ring.

The electron cloud effect, its impact on particle accelerator operation, and mitigation techniques have been studied in many laboratories worldwide but cannot be reviewed here. Recent development results and ongoing and proposed research work can be found in [1,2] and references therein. Possible mitigation techniques have been explored in the past, comprising grooved vacuum chambers, clearing electrodes, rough surfaces, and coatings such as amorphous carbon (a-C) and/or TiZrV.

The application of bare and enamel-coated stainless steel clearing electrodes to suppress the EC has been successfully demonstrated in the CERN PS $[3,4]$. The EC dynamics can also be changed by applying external electric, magnetic, or electromagnetic fields which is done, e.g., by the installation of solenoids along the beam pipe. However, accelerator bending sections can pose a limitation to this approach. In order to better control or even suppress the EC effect, the surface properties of the accelerator vacuum chambers play a crucial role, especially the SEY coefficient.

In order to evaluate the properties and efficiency of all different EC mitigation methods mentioned above, it is necessary to have experimental means for quantification. The goal is to measure the time dependence, the energy spectrum, and the density of the EC. Most experiments either use retarding field analyzers, button-type pickups, or stripline electrodes to detect the electrons and beam induced signals locally. The desired electron cloud density (ECD) can then be determined from these data. The ECD is assumed to be inhomogeneous over the entire length of the accelerator beam pipe. Therefore, local EC measurements have difficulties to detect these variations due to their limited transverse and longitudinal spatial resolutions.

A method to measure the integrated ECD over a longer section of an accelerator is the microwave transmission method, first introduced for CERN SPS electron cloud detection in 2003 [5] and developed further since then. This rather new technique measures the modulation of a microwave injected directly into the beam pipe. This modulation is caused by fluctuations of the ECD and therefore it is not limited to a local measurement but provides information of the EC over the whole measured section.

This paper describes the application on the microwave transmission method to study the physics of electron clouds in the SPS, and the EC suppression potential of carbon coated main dipole chambers, tested in 2009. 


\section{THEORETICAL BACKGROUND}

\section{A. Modulation}

Consider a purely sinusoidal wave with amplitude $A$ and frequency $\omega$ in the time domain:

$$
V(t)=A \cos (\omega t+\varphi)
$$

In order to transmit information, either the amplitude of this carrier wave $(\mathrm{CW})$ or its argument can be varied. Variation of the CW amplitude is referred to as amplitude modulation (AM), whereas the changing of the argument is called phase modulation (PM). Examples for AM and PM modulation are shown in Fig. 1. Detailed calculation of cases with sinusoidal modulations $\left[m(t)=\cos \left(\omega_{m} t\right)\right.$ for amplitude modulation, $m(t)=A_{m} \cos \left(\omega_{m} t\right)$ for phase modulation] can be found in [6]. For PM only narrowband modulation is considered in this paper. The results are

$$
\begin{aligned}
V_{\mathrm{AM}}(t)= & A_{\mathrm{C}} \cos \left(\omega_{\mathrm{C}} t\right)+\frac{\mathrm{a} A_{\mathrm{C}}}{2}\left\{\cos \left[\left(\omega_{\mathrm{C}}+\omega_{m}\right) t\right]\right. \\
& \left.+\cos \left[\left(\omega_{\mathrm{C}}-\omega_{m}\right) t\right]\right\},
\end{aligned}
$$
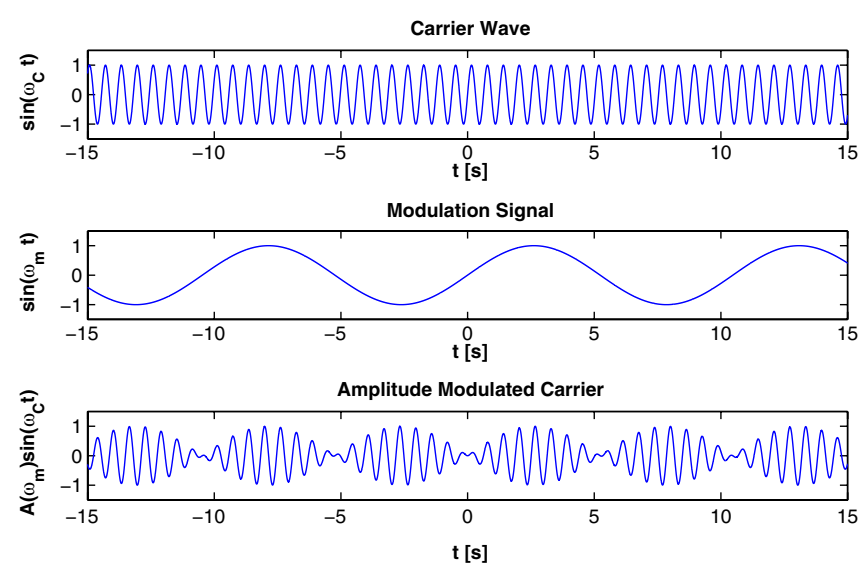

(a)
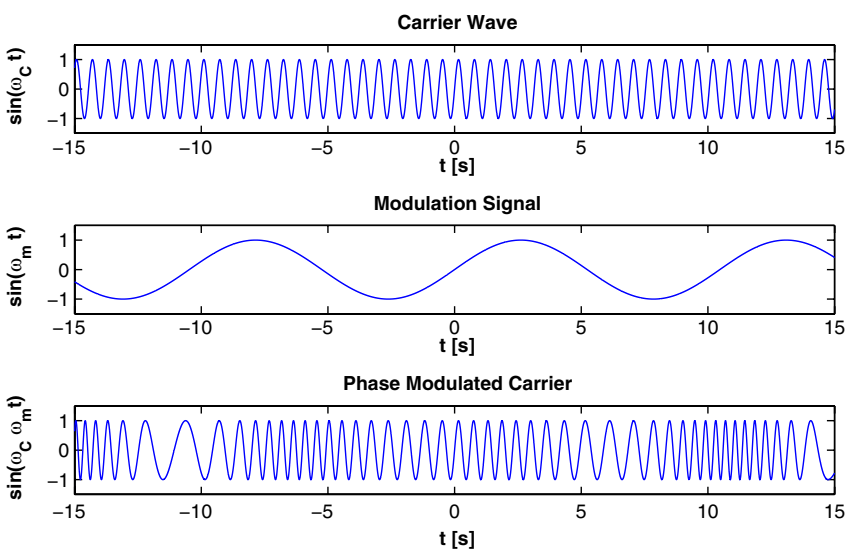

(b)

FIG. 1. Example for amplitude (a) and phase (b) modulation of a carrier wave.

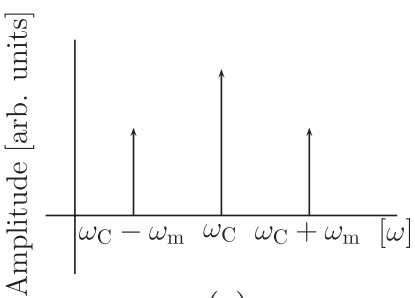

(a)

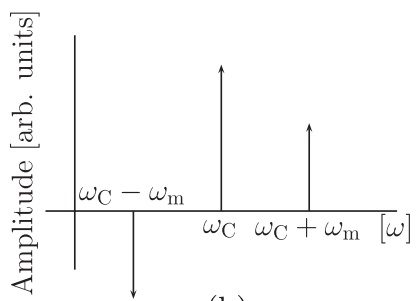

(b)
FIG. 2. Example for amplitude (a) and phase (b) modulation in frequency domain.

$$
\begin{aligned}
V_{\mathrm{PM}}(t)= & A_{\mathrm{C}} \cos \left(\omega_{\mathrm{C}} t\right)+\frac{\beta A_{\mathrm{C}}}{2}\left\{\cos \left[\left(\omega_{\mathrm{C}}+\omega_{m}\right) t\right]\right. \\
& \left.-\cos \left[\left(\omega_{\mathrm{C}}-\omega_{m}\right) t\right]\right\},
\end{aligned}
$$

where $A_{\mathrm{C}}$ is the amplitude of the unmodulated carrier, $\omega_{\mathrm{C}}$ is the frequency of the carrier wave, $\omega_{m}$ is the frequency of the modulation signal, and a, $\beta$ are modulation indices with $\beta=\frac{k_{f} A_{m}}{\omega_{m}}$ and $k_{f}$ as frequency deviation constant.

As can be seen comparing Eq. (2) and Eq. (3), the results of amplitude and narrowband phase modulation are almost identical. They only differ in sign, leading to a $180^{\circ}$ phase shift between PM and AM.

In the frequency domain, we get a spectrum with a sharp line at the center frequency $\omega_{\mathrm{C}}$ and lower lines $\left(\omega_{\mathrm{C}} \pm \omega_{m}\right)$, the so-called sidebands, due to the modulation. The principle is shown in Fig. 2. The sidebands are positioned left and right to the carrier at a distance corresponding to the frequency of the modulation signal. The phase shift between AM and PM can be clearly seen in Fig. 2 as well. Because of this phase shift, a combination of both modulation types results in different levels of the sidebands leaving the left sideband to be lower than the right one. Therefore, a mixture of AM and PM modulation can be recognized instantly by comparing the sideband levels.

\section{B. The principle of the microwave transmission method}

The basic principle of the microwave transition measurement is the analysis of the phase modulation an injected signal obtains when passing through a plasma (in this case the electron cloud). Figure 3 shows a schematic overview of a measurement setup illustrating the implementation of this method.

The resulting phase shift $\Delta \varphi$ over a length $L$ is the difference of the dispersion relation of an evacuated waveguide and the one in a waveguide filled with a homogeneous plasma [7], multiplied by the length:

$$
\Delta \phi=\Delta k L=\left[\left(\omega^{2}-\omega_{c}^{2}\right)^{1 / 2}-\left(\omega^{2}-\omega_{c}^{2}-\omega_{p}^{2}\right)^{1 / 2}\right] \frac{L}{c} .
$$

In the above equations $\omega$ is the injected frequency, $\omega_{c}$ is the cutoff frequency of the waveguide, $c$ is the speed of light, and $\omega_{p}$ is the plasma frequency. The latter is directly related to the integrated electron cloud density $n_{e}[8]$ via 


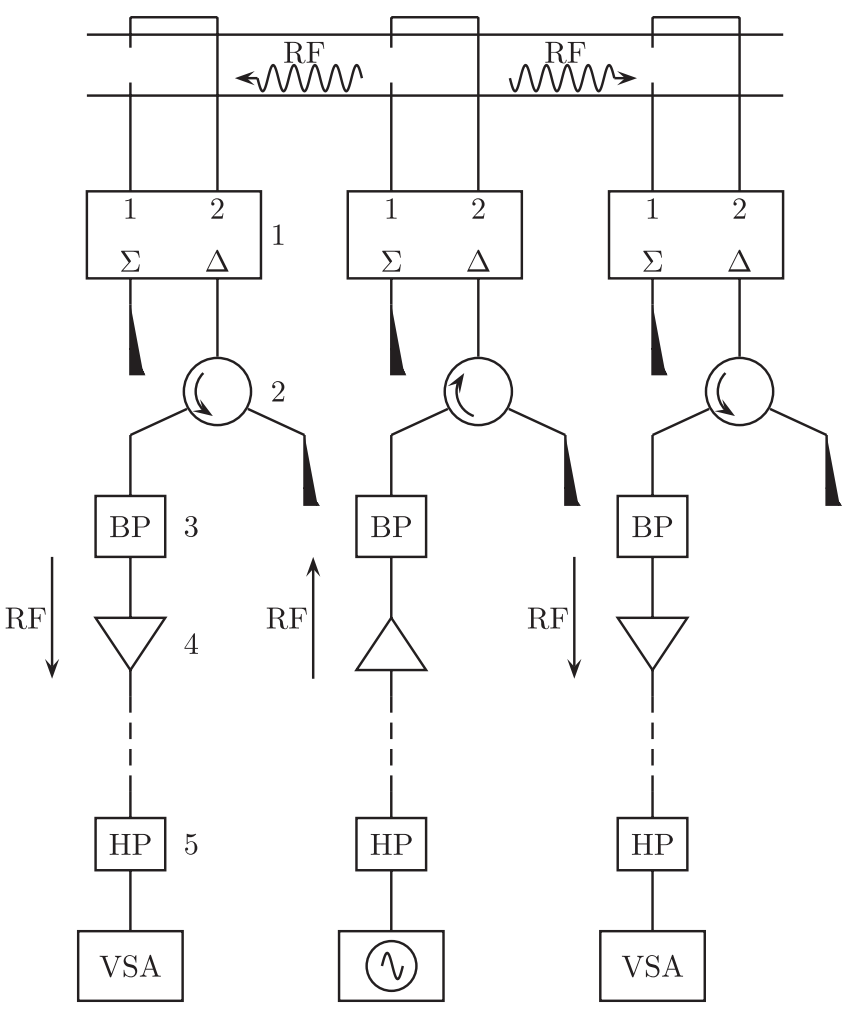

FIG. 3. Schematic layout of the SPS microwave transmission measurement. Each path consists of a hybrid (1), a circulator (2), a band-pass filter (3), an amplifier (4), and a high pass (5). The amplifier was powered by a Hameg Triple Power Supply HM 7042-5.

$$
\omega_{p}=\sqrt{\frac{n_{e} e^{2}}{\epsilon_{0} m_{e}}} \cong 56.4 \sqrt{n_{e}}
$$

where $e$ is the electron charge, $\epsilon_{0}$ is the permittivity in free space, and $m_{e}$ is the electron mass.

A linear expansion of Eq. (4) for small $\omega_{p}$ leads to the following relation for the phase shift [8]:

$$
\Delta \varphi \approx \frac{L \omega_{p}^{2}}{2 c\left(\omega^{2}-\omega_{c}^{2}\right)^{1 / 2}}
$$

Note that formula (6) is only valid for a neutral plasma but corrections for the charge are very small and in our case negligible as shown in simulations [8].

The cyclotron resonance at a frequency of $28 \mathrm{GHz} / \mathrm{T}$ times the dipole field strength [9] would be $3.3 \mathrm{GHz}$ at flat bottom for the magnetic cycle of the SPS, which was confirmed in previous measurements [10]. Since this value is well above the chosen $\mathrm{CW}$ frequency, this resonance is not excited in this setup.

\section{EXPERIMENTAL SETUP}

In March 2009 the microwave transmission experiment was set up in sector 5 of the CERN SPS between two dipole
TABLE I. Parameters of the experimental setup and the CERN SPS.

\begin{tabular}{lc}
\hline \hline Injected frequency $\omega$ & $f=2.68 \mathrm{GHz}$ \\
Cutoff frequency $\omega_{c}$ & $f=2.4 \mathrm{GHz}$ \\
Transmission length $L$ & $6.5 \mathrm{~m}$ \\
SPS revolution frequency & $43.35 \mathrm{kHz}$ \\
Bunch spacing LHC nominal beam & $24.97 \mathrm{~ns}$ \\
Batch spacing for 25 ns beam & $224.7 \mathrm{~ns}$ \\
SPS circumference & $6911 \mathrm{~m}$ \\
Magnetic field strength at flat bottom & $0.117 \mathrm{~T}$ \\
\hline \hline
\end{tabular}

magnets, one at position 51470 and the other at position 51490. Both MBB-type magnets comprise a vacuum chamber of $132 \mathrm{~mm}$ horizontal and $51.5 \mathrm{~mm}$ vertical aperture. The vacuum chamber of magnet 51490 was coated with a layer of amorphous carbon of 200-300 nm thickness. The microwave signal was coupled into the vacuum chamber via a coupling loop located between both magnets. The signal pickups were installed upstream and downstream of these magnets at a distance of $6.5 \mathrm{~m}$ from the center. A schematic overview of the experimental setup is depicted in Fig. 3.

To transfer the stimulus signal into the SPS tunnel, a signal generator (Rhode \& Schwarz SM 300 Signal Generator $9 \mathrm{kHz}-3 \mathrm{GHz}$ ) was used. This generator has an adjustable internal amplifier which has been set to a level of $13 \mathrm{~dB}$ for the majority of the measurements. For many measurements, an artificial modulation signal created by the generator was injected together with the carrier wave as a calibration signal. All signals were injected into the central loop antenna of our setup (Fig. 3), giving the same physical distance after passing through the uncoated beam pipe of MBB 51470 and after passing through the coated beam pipe of MBB 51490.

The analysis and visualization of the measurements were done with a vector spectrum analyzer (VSA, Agilent Technologies MXA Signal Analyzer) using the demodulation function for amplitude as well as phase demodulation.

All further parameters of the experimental setup in the SPS are summarized in Table I.

\section{Coupling}

In principle, the best mode for coupling would be the $\mathrm{H}_{10}$ mode which has a cutoff frequency of $1.23 \mathrm{GHz}$. However, the hardware transfer function (HTF) [11] of the experimental setup is very low in this frequency range and practically no coupling could be achieved. Since the HTF has a maximum at $2.68 \mathrm{GHz}$ testing showed that the coupling at this frequency was optimal. Being above the cutoff of the $\mathrm{H}_{20}$ mode of $2.4 \mathrm{GHz}$, coupling to this mode was chosen. An overview of the modes up to $3 \mathrm{GHz}$ is given in Table II. 
TABLE II. Modes and their cut off frequency in the vacuum chamber of CERN SPS.

\begin{tabular}{lc}
\hline \hline Mode & Frequency [GHz] \\
\hline $\mathrm{H}_{10}$ & $1.23 \mathrm{GHz}$ \\
$\mathrm{H}_{20}$ & $2.4 \mathrm{GHz}$ \\
$\mathrm{H}_{11}$ & $2.95 \mathrm{GHz}$ \\
$\mathrm{E}_{11}$ & $3.09 \mathrm{GHz}$ \\
$\mathrm{H}_{21}$ & $3.36 \mathrm{GHz}$ \\
\hline \hline
\end{tabular}

\section{RESULTS}

All measurements were performed in the frequency domain, focusing the frequency range to observe the relevant effects, e.g., the carrier wave frequency of $2.68 \mathrm{GHz}$, and the first modulation sidebands caused by the electron cloud. The sidebands are located at integer multiples of the SPS revolution frequency $(43.347 \mathrm{kHz})$; their amplitude decreases in level with distance to the carrier.

\section{A. Comparison between coated and uncoated MBB dipole vacuum chambers}

A clear difference of the measured phase modulation (PM) signal in the coated and uncoated section was seen in
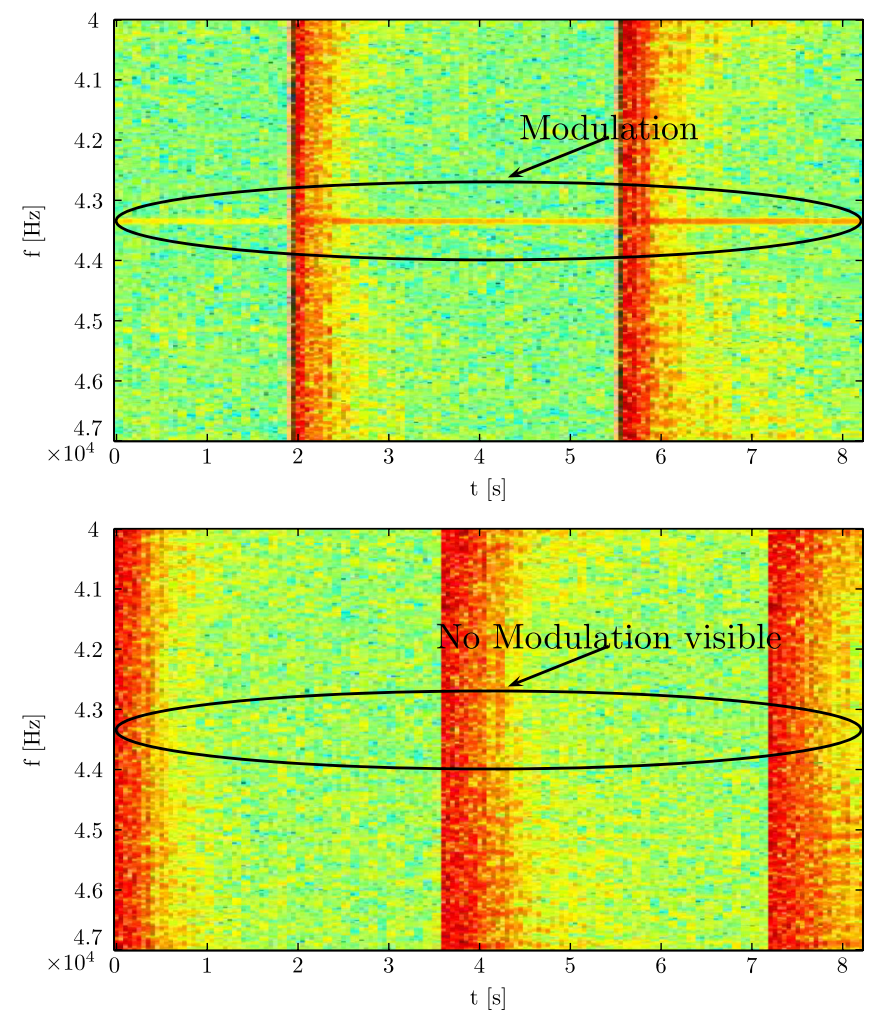

FIG. 4. Spectrum mode after phase demodulation in the uncoated (upper graph) and coated (lower graph) section. The red peaks correspond to the injection of a new batch. The data were taken with the nominal LHC 25 ns beam of four batches at SPS flat bottom.

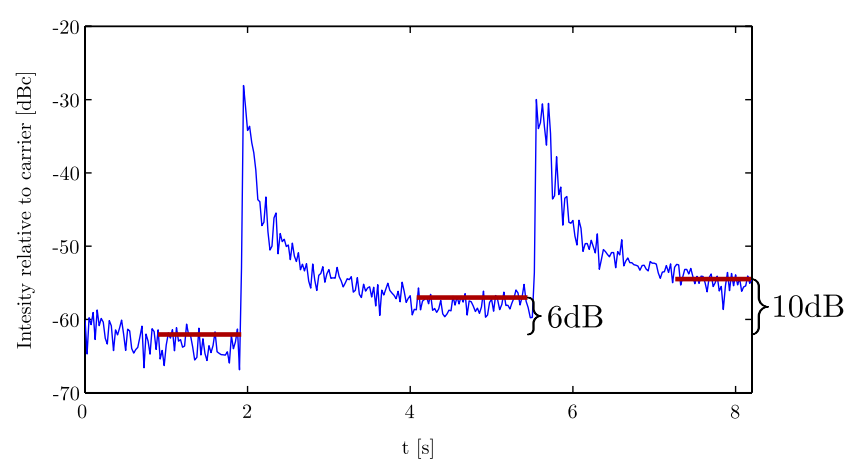

(a)

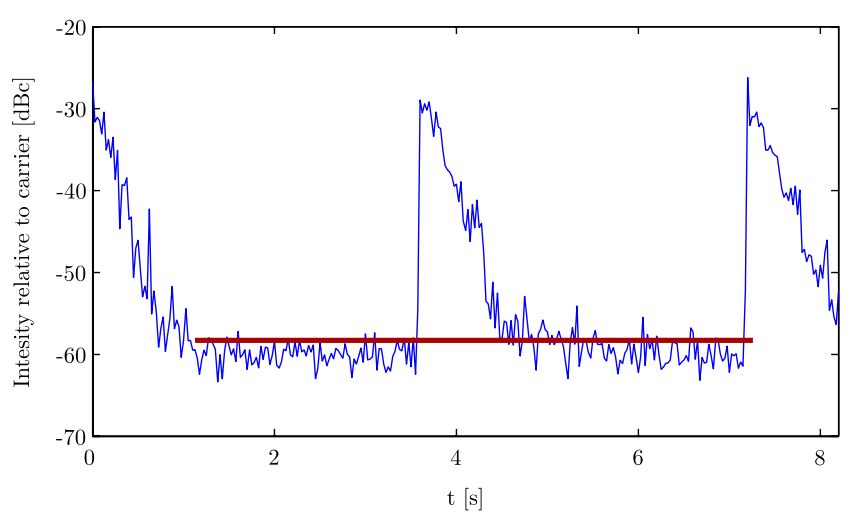

(b)

FIG. 5. Phase modulation signal over time in the uncoated (a) and coated (b) section. The peaks in the graph correspond to the injection of a new batch. The data were taken with the nominal LHC $25 \mathrm{~ns}$ beam of four batches at SPS flat bottom.

the machine development (MD) run in September 2009. In the uncoated section, a signal was visible (on average $6 \mathrm{~dB}$ over the noise floor), while in the coated section no signal could be resolved above the noise floor (Fig. 4). Therefore, the signal in the coated section is at least a factor 2 lower. The explicit development of the signal trace over time is shown in Fig. 5. There the total increase of the signal over time can be seen in the uncoated case, whereas in the coated section the signal decreases to the noise floor level shortly after injection.

These results could be confirmed nicely during an MD run in November 2009.

\section{B. Calibration signal and electron cloud monitor}

During the MD run in November 2009, a purely phase modulated signal was artificially created with the signal generator (at $42 \mathrm{kHz}$ off center frequency $f=2.68 \mathrm{GHz}$ ) and sent down together with the carrier signal as calibration signal (Fig. 6). A signal modulation of $0.1 \mathrm{rad}$ (roughly a factor 100 higher than the expected signal) was chosen in order to ensure its detection. Thus, the status of the electronics chain is monitored over the complete cycle, in which we also observed saturation effects (Fig. 7). Since 

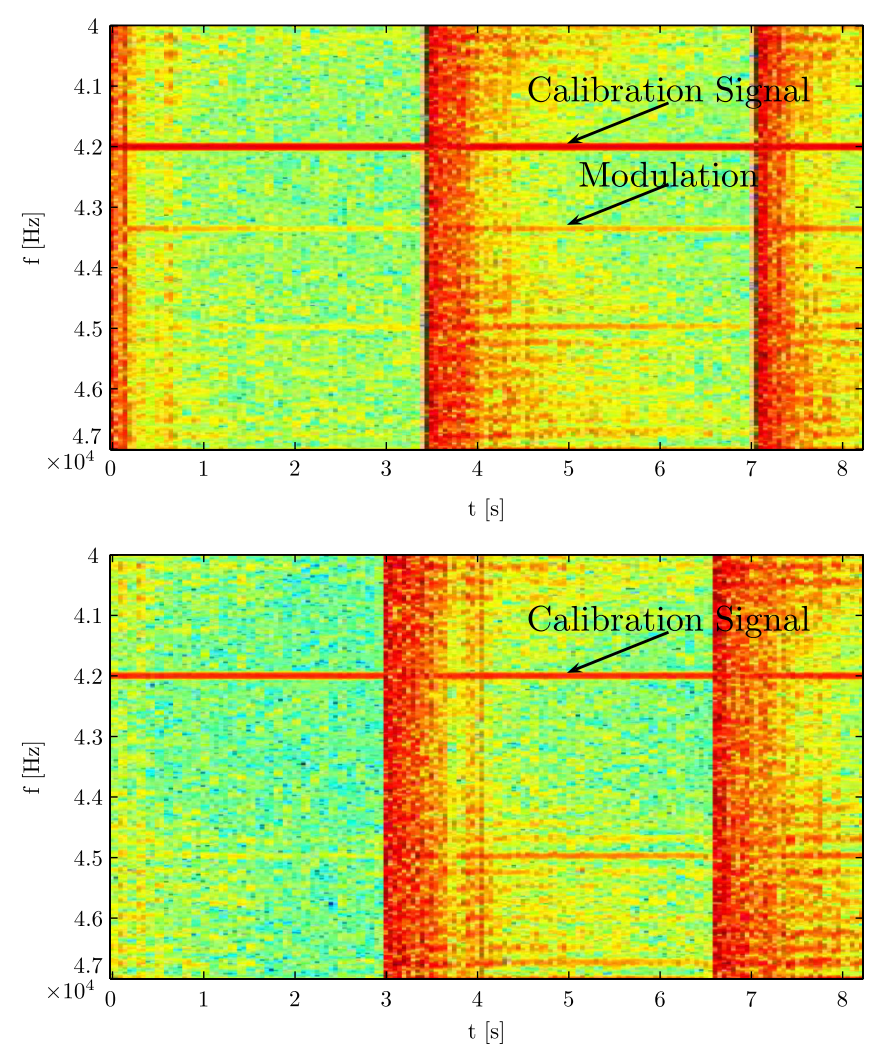

FIG. 6. Spectrum after phase demodulation. The calibration line can be seen in the coated as well as in the uncoated section whereas the genuine modulation line at around $43 \mathrm{kHz}$ can only be seen in the uncoated case. The data were taken with the nominal LHC $25 \mathrm{~ns}$ beam of four batches at SPS flat bottom.

the calibration signal is artificial and hence independent of the accelerator, it should not be related to the machine cycle. Nevertheless, a slight ripple in the signal at each injection was observed. This was most likely a saturation effect in our electronics. After the ripple in the calibration

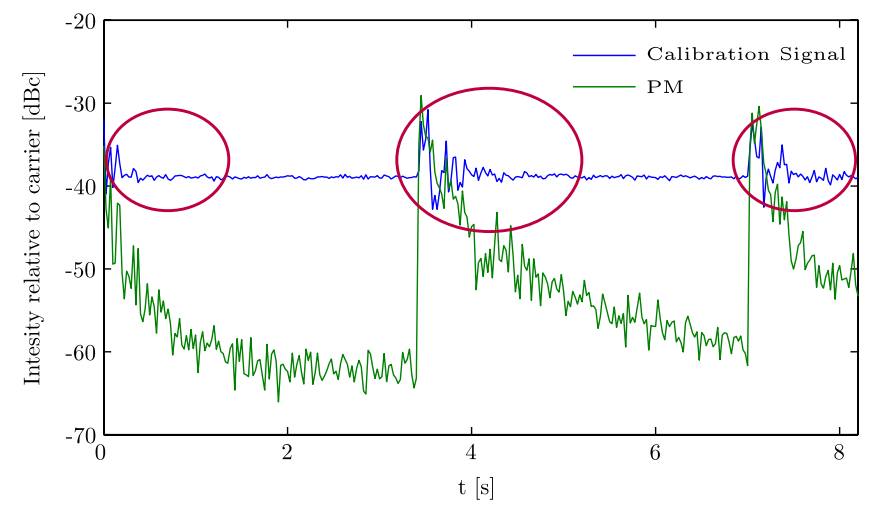

FIG. 7. Graph of the calibration signal after phase demodulation. The red circles show an interval, after which the measured signal can be considered reliable, since no saturation effects in the electronic chain are indicated. The data were taken with the nominal LHC $25 \mathrm{~ns}$ beam of four batches at SPS flat bottom.

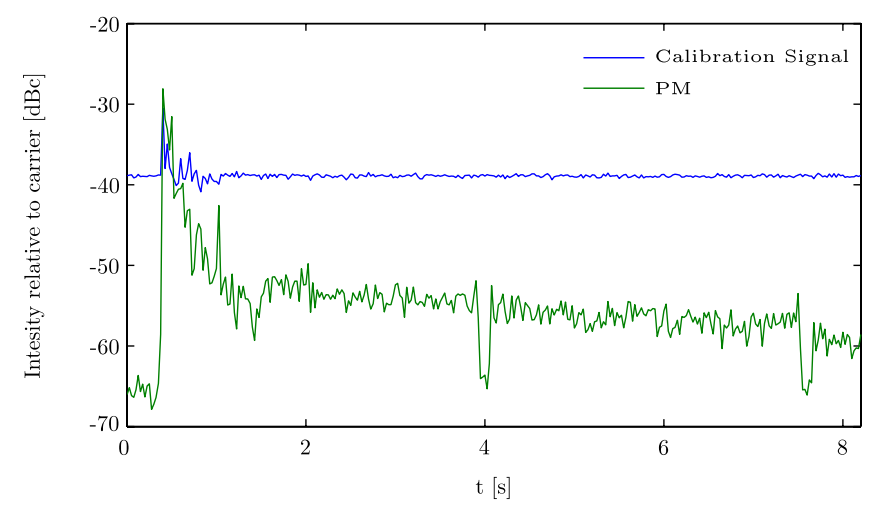

FIG. 8. Time development of the PM signal compared to a calibration signal after phase demodulation. The data were taken with the nominal LHC $25 \mathrm{~ns}$ beam of one batch at SPS flat bottom.

signal had disappeared, a more or less smooth line remained, indicating an interval in which the measurement signal was not perturbed by nonlinear effects in our electronic chain. Hence, confidence intervals for our measurements could be determined (Fig. 7). As shown in Fig. 8, the calibration line (blue) was nearly flat, as it should be, whereas glitches in the beam cycle (green measurement signal) could be observed. Therefore it can be deduced that the latter signal is related to the machine cycle, whereas the artificial signal indeed does not depend on it. A comparison of the data from the microwave transmission measurements to the data obtained with the electron cloud monitor showed an excellent agreement between both methods (Fig. 9). In both cases a signal increase after each injection followed by a decay can be clearly seen [Fig. 9(a)]. Also the comparison of cases with programmed short term changes of the beam structure appearing as dips in the electron cloud signal illustrates the correlation of both methods nicely [Fig. 9(b)].

\section{Different beam types}

In November 2009, the SPS microwave transmission setup was tested with different LHC-type beams, namely, with 25, 50, and $75 \mathrm{~ns}$ bunch spacing. The electron cloud density should be highly reduced at a $50 \mathrm{~ns}$ bunch spacing and no electron cloud should occur in the case of $75 \mathrm{~ns}$ bunch spacing, since there is enough time for it to decay between the batches $[12,13]$. This could be confirmed with our measurement method.

For the uncoated vacuum chamber, a comparison between 25 and $50 \mathrm{~ns}$ spacing shows that the signal level is substantially lower for the $50 \mathrm{~ns}$ beam $(6 \mathrm{~dB}$ vs $1 \mathrm{~dB}$ after first injection, $10 \mathrm{~dB}$ vs $2 \mathrm{~dB}$ after second injection). For the 75 ns beam no signal was observed (Fig. 10).

In the case of the coated vacuum chamber we did not observe any sideband signals, independent of the LHC beam type. At $75 \mathrm{~ns}$ beamspacing even the injections were invisible, thus, under these conditions the electronics 

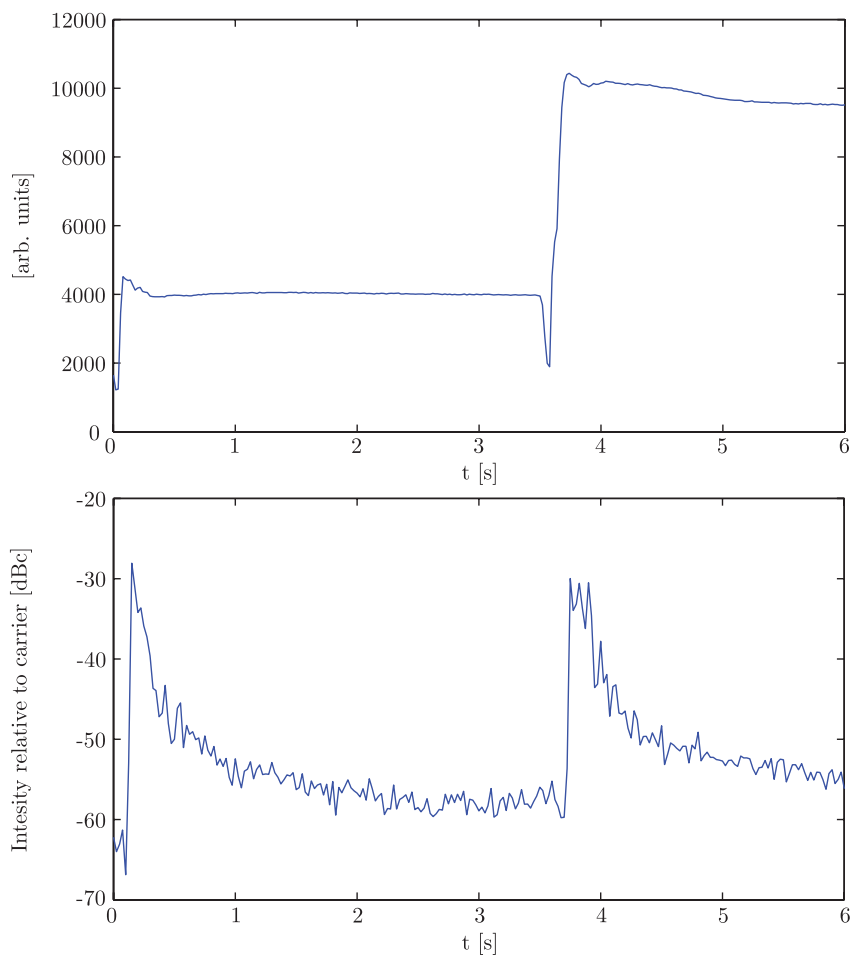

(a)
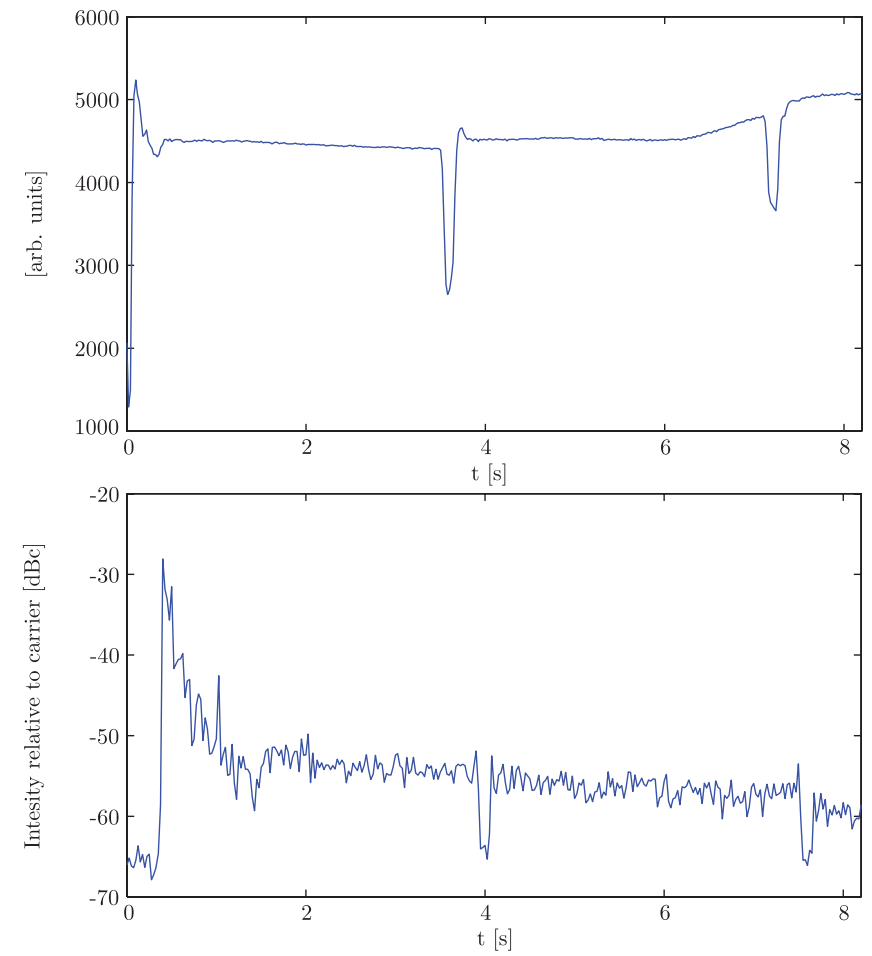

(b)

FIG. 9. Comparison of the phase modulation signal from the microwave transmission experiments [lower graph in (a) and (b)] to the electron cloud monitor readings [upper graph in (a) and (b)]. In (a), the data was taken with the nominal LHC 25 ns beam of four batches at SPS flat bottom. In (b), the data was taken with the nominal LHC $25 \mathrm{~ns}$ beam of one batch at SPS flat bottom. Dips in the signal are seen with both methods.
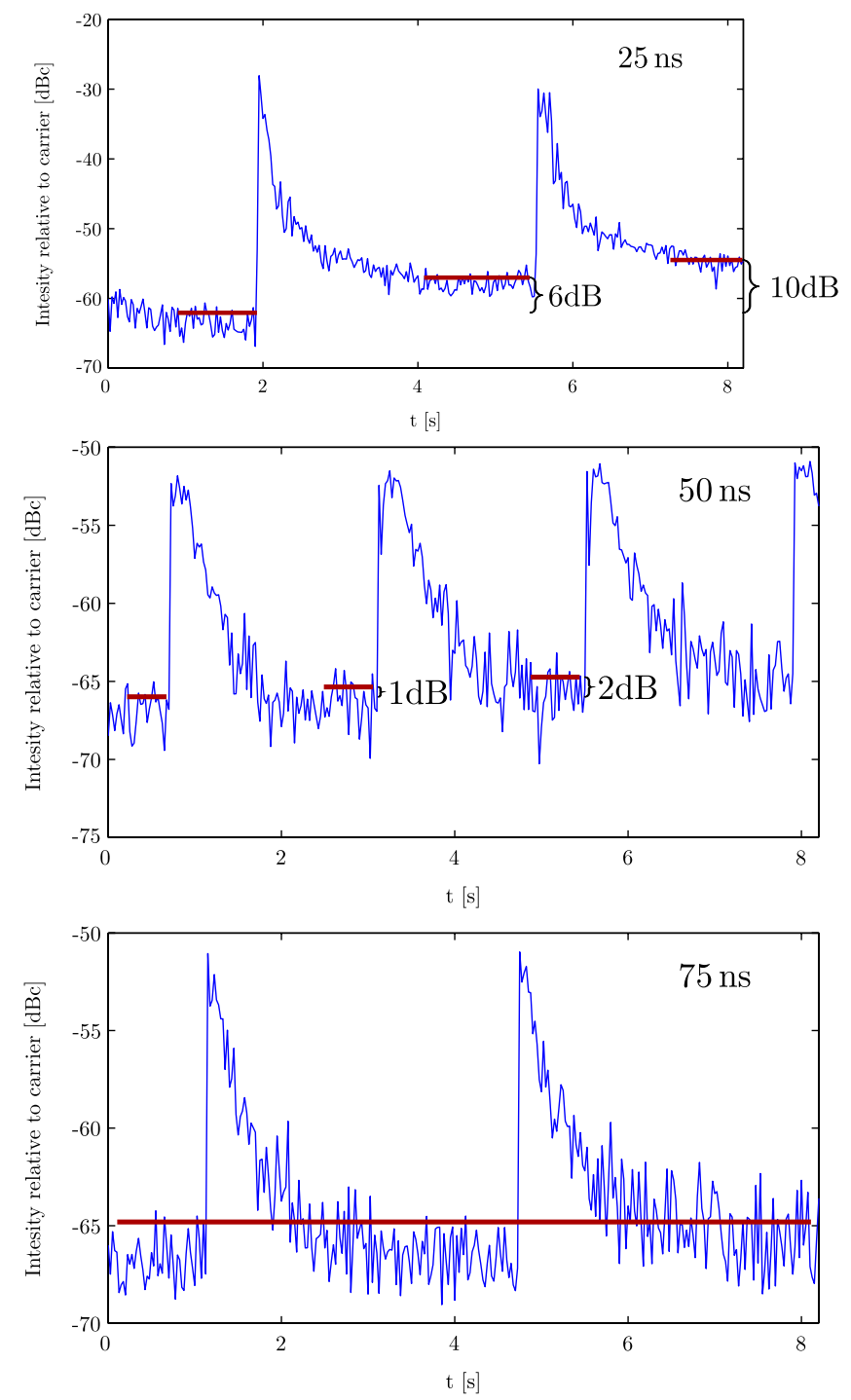

FIG. 10. Comparison of phase modulation signals, measured for the uncoated SPS vacuum chamber, using the nominal LHC beams with 25, 50, and $75 \mathrm{~ns}$ bunch spacing. The data were taken with beams of four batches at SPS flat bottom.

appear to operate in the linear regime-no saturation effects (Fig. 11). These obtained microwave transmission results are in good agreement with the electron cloud monitor readings.

\section{Quantitative evaluation}

For a quantitative evaluation, one has to keep in mind that the measured phase shift is related to the integrated electron cloud density, thus, it is an average over buildup, plateau value, and decay of the electron cloud. Since only the plateau value is of interest, the duty cycle of the SPS machine has to be taken into account and corrected for. The averaging over the circumference should not be confounded with the signal averaging over several turns which leads to a reduction of the signal amplitude. 

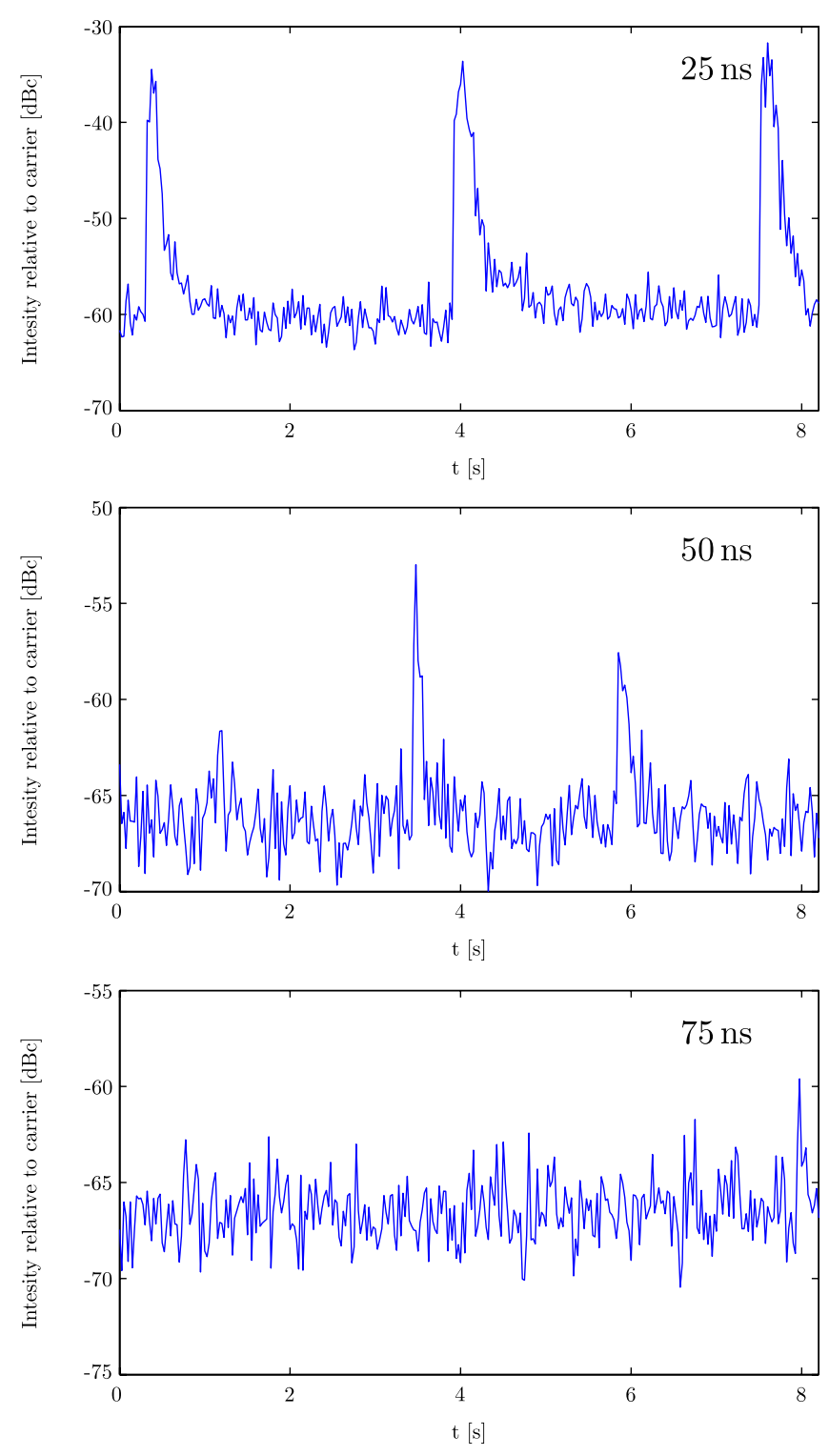

FIG. 11. Comparison of phase modulation signals, measured for the coated SPS vacuum chamber, using the nominal LHC beams with 25, 50, and $75 \mathrm{~ns}$ bunch spacing. No signal is seen for any bunch spacing. For the $75 \mathrm{~ns}$ beam not even an injection signal is visible. The data were taken with beams of four batches at SPS flat bottom.

For the nominal LHC beam in the SPS, the batches are spaced by $\approx 225 \mathrm{~ns}$ and each batch consists of 72 bunches equally spaced with 25 ns. Simulations of the electron cloud distribution for this beam type show that the peak value of the electron cloud density lasts for $1 \mu \mathrm{s}$ over the first batch and $\approx 1.6 \mu$ s for each following batch (Fig. 12). The duty cycle of the machine, which is the ratio of the part of the machine occupied by the batches (bunch trains) to the total circumference, can be determined by relating the duration of the electron cloud signal of one turn to the machine circumference. For one batch this is

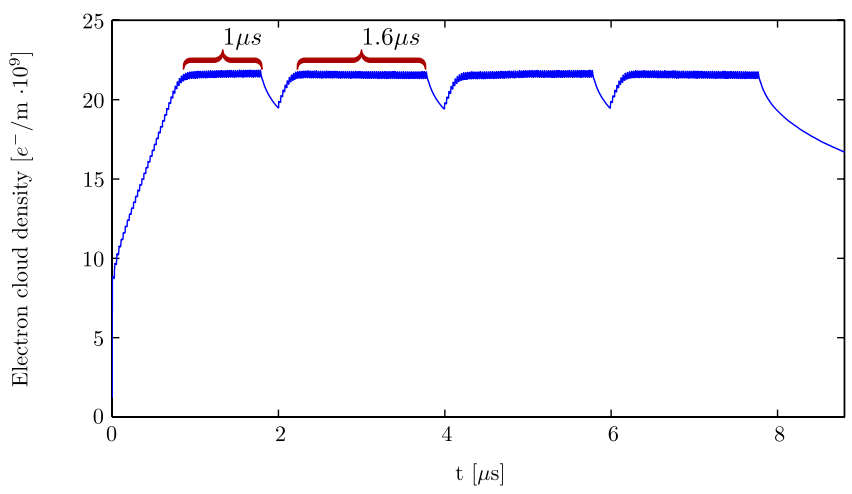

FIG. 12. Electron cloud simulation results (MBB-type SPS vacuum chamber) for the nominal LHC 25 ns beam (courtesy G. Rumolo).

$$
\text { duty cycle }=\frac{1 \times 10^{-6} \mathrm{~s}}{6911 \mathrm{~m} / 3 \times 10^{8} \mathrm{~ms}^{-1}}=4.34 \% \text {. }
$$

The results for two, three, and four batches are listed in Table III. The maximal variation and, hence, the maximal signal from the electron cloud occurs for a $50 \%$ duty cycle. Since the duty cycles in the SPS are much lower than that, the measured values for each beam are too low. They have to be corrected as only the maximum value is of interest since formula (6) refers to the peak value, assuming a completely filled machine. To facilitate the determination of the correction factors, it can be assumed that the electron cloud signal has a rectangular shape. Decomposing this shape for the different cycles into its Fourier coefficients, the fundamental modes (i.e. the first Fourier coefficient) can be related to the fundamental mode of the $50 \%$ cycle. The differences can be expressed in $\mathrm{dB}$, and hence used directly for correction of the measured signal. The calculation of the first Fourier coefficients can be done quite easily for all forementioned duty cycles using, e.g., online tools for Fourier analysis [14].

The correction factors for the different beams are summarized in Table IV. They have to be added to the measured integrated ECD. To determine whether the assumption of a rectangular signal shape is a good approximation, calibration measurements have been performed as follows.

The signal generator was directly connected to the vector spectrum analyzer and a $\mathrm{CW}$ signal of $100 \mathrm{MHz}$ as well

TABLE III. Duty cycle in percent for different beams in the SPS.

\begin{tabular}{lc}
\hline \hline Number of batches & Duty cycle [\%] \\
\hline 1 batch & 4.34 \\
2 batches & 11.3 \\
3 batches & 18.23 \\
4 batches & 25.2 \\
\hline \hline
\end{tabular}


TABLE IV. Correction factors for different beams in the SPS.

\begin{tabular}{lc}
\hline \hline Number of batches & Correction factor [dB] \\
\hline 1 batch & 17.4 \\
2 batches & 9.3 \\
3 batches & 5.5 \\
4 batches & 2.9 \\
\hline \hline
\end{tabular}

TABLE V. Comparison of measured and calculated correction factors for different duty cycles.

\begin{tabular}{ccc}
\hline \hline $\begin{array}{l}\text { Duty } \\
\text { cycle [\%] }\end{array}$ & $\begin{array}{c}\text { Measured correction } \\
\text { factor [dB] }\end{array}$ & $\begin{array}{c}\text { Calculated correction } \\
\text { factor [dB] }\end{array}$ \\
\hline 40 & 0 & 0.47 \\
30 & 1 & 1.8 \\
20 & 4 & 4.6 \\
\hline \hline
\end{tabular}

as a modulation signal of $0.01 \mathrm{rad}$ was generated and analyzed in PM demodulation. The signal generator, however, was triggered externally by a rectangular modulation created by an external generator (Hewlett Packard 33120A $15 \mathrm{MHz}$ function/arbitrary waveform generator). Different duty cycles were simulated by variation of the pulse duration of the rectangular signal. The maximum value was found at a 50\% duty cycle as expected. Comparison of the measured correction factors, obtained by relating the duty cycles to the maximum value, and the calculated values show a good agreement (Table V). Adding the calculated correction factors to the measured signals and using the calibration signal height to determine the phase shift, the ECD can be estimated using formula (6). For the LHC $25 \mathrm{~ns}$ beam a value of $1.5 \times 10^{12} \mathrm{~m}^{-3}$ is found for the ECD.

\section{SUMMARY AND OUTLOOK}

The microwave transmission method proved to be a valuable tool for electron cloud detection. The mitigation properties of the amorphous carbon coating applied in the SPS main dipole chambers could be tested successfully. The integrated electron cloud density was proven to be at least $6 \mathrm{~dB}$ lower in the coated vacuum chamber and, hence, a beneficial effect of this coating was confirmed. Comparison to the electron cloud monitor showed an excellent agreement between the two methods. The reduction of the electron cloud density for larger bunch spacings, as predicted by simulations, could be confirmed. The electron cloud density was evaluated to be $1.5 \times 10^{12} \mathrm{~m}^{-3}$ (SPS MBB-type vacuum chamber) which is in good agreement with simulations. In 2010, experiments will continue in the SPS with the microwave transmission over a pair of coated MBB dipole vacuum chambers, resulting in a doubling of the transmission length. This is expected to increase the modulation signal and, hence, to increase the sensitivity limit of this method by nearly the same factor.

\section{ACKNOWLEDGMENTS}

First of all, it is a pleasure to thank T. Kroyer and D. Seebacher for their help to further develop the SPS microwave transmission technique. Special thanks also to G. Rumolo, B. Salvant, M. Taborelli, and C. Yin Vallgreen for many fruitful discussions. Suggestions and comments from members of the SPS upgrade study team are appreciated very much as well as the technical help received from the CERN TE-VSC and TE-MSC groups. The continuous support from E. Ciapala, E. Shaposhnikova, and J. M. Jimenez is highly acknowledged.

[1] Proceedings of Electron Cloud Mitigation (ECM08) Workshop, CERN.

[2] Proceedings of Anti e-Cloud Coatings (AEC09) Workshop, CERN.

[3] E. Mahner, T. Kroyer, and F. Caspers, Phys. Rev. ST Accel. Beams 11, 094401 (2008).

[4] F. Caspers, T. Kroyer, E. Mahner, and J. Wendel, in Proceedings of the 23rd Particle Accelerator Conference, Vancouver, Canada, 2009 (IEEE, Piscataway, NJ, 2009).

[5] T. Kroyer, F. Caspers, and E. Mahner, in Proceedings of the 21st Particle Accelerator Conference, Knoxville, 2005 (IEEE, Piscataway, NJ, 2005), p. 2212.

[6] R.A. Witte, Spectrum and Network Measurements (Prentice Hall Inc., Englewood Cliffs, NJ, 1991).

[7] H. S. Uhm, K. T. Nguyen, R. F. Schneider, and J. R. Smith, J. Appl. Phys. 64, 1108 (1988), http: //link.aip.org/link/? JAP/64/1108/1.

[8] K. Sonnad, M. Furman, S. Veitzer, P. Stoltz, and J. Cary, in Proceedings of the 2007 Particle Accelerator Conference, Albuquerque, New Mexico (IEEE, Albuquerque, New Mexico, 2007).

[9] F. Caspers and F. Zimmermann, in Proceedings of the 23rd Particle Accelerator Conference, Vancouver, Canada, 2009 (Ref. [4]).

[10] T. Kroyer, F. Caspers, W. Hoefle, J. M. Jimenez, J.-F. Malo, and J. Tueckmantel, in Proceedings of the 31st ICFA Advanced Beam Dynamics Workshop (ECLOUD 04) (2005) [http://accelconf.web.cern.ch/accelconf/e96/ PAPERS/MOPG/MOP085G.PDF].

[11] H. Stockhorst, U. Bechstedt, J. Dietrich, R. Maier, S. Martin, D. Prasuhn, A. Schnase, H. Schneider, and R. Tolle, in Proceedings of EPAC 96 (1997), Vol. 1, p. 1048 [http://accelconf.web.cern.ch/accelconf/e96/PAPERS/ MOPG/MOP085G.PDF].

[12] G. Arduini, https://ab-div.web.cern.ch/ ab-div/Conferences/ Chamonix/chamx2004/PAPERS/1_02_AG.pdf.

[13] G. Rumolo, https://paf-spsu.web.cern.ch/paf-spsu/meetings/ 2007/M20-11/GR-SPSU5.pdf.

[14] http://www.eecircle.com/applets/001/001.html. 